\title{
¿CUÁles SON LOS CRITERIOS DE VALORACIÓN EN SALUd LABORAL?
}

\author{
Rosa Montero Simó \\ Consejería de Empleo y Desarrollo Tecnológico \\ Junta de Andalucía
}

La intención de la autora en este artículo es orientar al especialista en Derecho sobre el significado que tienen los criterios de valoración para agentes químicos, físicos y biológicos, utilizados como referencia por los técnicos en Prevención de Riesgos Laborales para el estudio y protección de la salud del trabajador en su lugar de trabajo.

Se exponen cuales son los valores legales actuales asi como los que se emplean habitualmente en aquellos campos que carecen de normativa, basándose en bibliografía científica contrastada.

In this article the author seeks to give advice to Labour Relations Lawyers on the meaning of occupational exposure standards for chemical, physical and biological agents. These criteria are used as a reference by Occupational Safety and Health Experts in the study and protection of workers' health at their workplace.

Both the present legal criteria and the ones used if there is no set of rules available, will be explained in this article on the basis of a contrastive scientific bibliography.

Palabras clave: Criterio de Valoración. Agentes Químicos. Agentes Físicos. Agentes Biológicos. Normativa Legal. Salud Laboral

Key words: Occupational Exposure Standards. Chemical Agents. Physical Agents. Biological Agents. Legal Rules. Occupational Safety and Healtb

\section{INTRODUCCIÓN}

En Higiene Industrial se entiende por criterio de valoración los parámetros contenidos en la norma, tanto legal como técnica, con la que se comparan los resultados conseguidos tras el muestreo de agentes químicos, físicos ó biológicos en el ambiente de trabajo, con ello se obtiene información del riesgo que puede entrañar el contaminante para la salud de los trabajadores. 
Hablamos de norma legal porque contamos con una serie de reglamentos que contienen dichos criterios de valoración y en el caso de que nos encontremos con un vacío, al no tener parámetros legales con los que comparar, podemos emplear normas técnicas, UNE, EN, ISO y bibliografía, emitida por instituciones de reconocida solvencia, que empleamos como referencia.

$\mathrm{Al}$ realizar la evaluación de los agentes químicos, físicos y biológicos en el lugar de trabajo se obtiene la concentración o nivel de los mismos, que junto con el tiempo de contacto de los trabajadores con ellos configuran lo que entendemos por exposición. También han de tenerse en cuenta hábitos personales, métodos de trabajo, etc. La comparación de la exposición con el criterio de valoración define el riesgo para la salud según ese criterio de valoración.

Los distintos valores propuestos como criterios de valoración se basan en información obtenida a partir de diversas fuentes: estudios epidemiológicos, toxicológicos con animales, químico-toxicológicos, ensayos con voluntarios, etc. El diseño de un criterio de valoración implica la definición de dos cuestiones básicas relacionadas entre sí:

- Que efecto máximo sobre la salud se establece como admisible.

- Que porcentaje de la teórica población expuesta se está realmente protegiendo.

El efecto máximo sobre la salud que se admite cuando se establece el criterio implica un valor de dosis máxima tolerable o admisible. Una vez disponemos de él y admitiendo condiciones de trabajo estándar, se proponen los valores límite estimados a través de una relación entre la concentración o nivel y la dosis.

Los criterios de valoración, excepto en los casos en los que se trate de un reglamento que haya de cumplirse, deben tomarse como una referencia orientativa de la medida efectuada. Nunca deben considerarse como una frontera entre condiciones seguras $y$ peligrosas y cuando se proceda a su aplicación deberán tenerse en cuenta los factores que puedan modificar la exposición réal al contaminante. 
2. CRITERIOS DE VALORACión Para agentes Químicos

2.1. EN EL AIRE

\subsubsection{Normativa interna Española}

La normativa interna española sobre agentes químicos y las concentraciones ambientales de los mismos en lugares de trabajo está integrada por distintas fuentes.

En primer lugar, el Decreto 2414/1961, de 30 de septiembre, por el que se aprueba el Reglamento de Actividades Molestas, Insalubres, Nocivas y Peligrosas $^{1}$, RAMINP, en cuyo ANEXO II aparece una lista de 160 sustancias químicas, para las que se fija una "concentración máxima permitida", sin otras especificaciones.

En segundo lugar, la Resolución de 15 de febrero de $1977^{2}$, de las Direcciones Generales de Trabajo y Promoción industrial y Tecnología, por la que se actualizan las instrucciones complementarias de desarrollo de la Orden de Presidencia de Gobierno de 14 de septiembre de 1959 que regula el empleo de disolventes y otros compuestos que contengan Benceno ${ }^{3}$.

\section{- BENCENO}

Concentración máxima permitida $25 \mathrm{ppm}-80 \mathrm{mg} / \mathrm{m}^{3}$

En tercer y último lugar, todos los reglamentos derivados de transposición de Directivas Europeas, en los que aparecen valores límite:

Orden de 9 de abril de 1986, por la que se aprueba el Reglamento para la prevención de riesgos y protección de la salud por la presencia de Cloruro de Vinilo Monómero en el ambiente de trabajót.

- CLORURO DE VINILO MONÓMERO

Concentración promedio anual

Concentración promedio diaria
$3 \mathrm{ppm}$

$7 \mathrm{ppm}$

1. $\mathrm{BOE} 7-12-61$ y $7-3-62$

2. BOE 11-3-1977

3. BOE 18-9-1959

4. BOE 6-5-1986 
Orden de 9 de abril de 1986, por la que se aprueba el Reglamento para la prevención de riesgos y protección de la salud de los trabajadores por la presencia de Plomo Metálico y sus compuestos iónicos en el ambiente de trabajo5.

- PLOMO METÁLICO Y SUS COMPUESTOS IÓNICOS

Concentración promedio diaria $\quad 0.15 \mathrm{mg} / \mathrm{m}^{3}$

Real Decreto 88/1990, de 26 de enero, sobre protección de los trabajadores mediante la prohibición de determinados agentes especificos o determinadas actividades ${ }^{6}$.

- PROHIBICIÓN DE AGENTES O ACTIVIDADES

Se prohibe la producción y utilización de:

2-naftilamina y sus sales

Bencidina y sus sales

4-aminobifenilo y sus sales

4-dinitrofenilo

Excepto si los agentes son impurezas, subproductos o elementos de desecho de otras sustancias o preparados, siempre que su concentración sea inferior al $0.1 \%$ en peso.

Quedan exceptuadas las siguientes actividades y procesos:

1. Investigación y experimentación, incluido análisis.

2. Procesos para eliminar estos agentes.

3. Procesos en que los agentes aparezcan como productos intermedios.

Orden de 16 de octubre de 1991, por la que se aprueba la Instrucceión Técnica Complementaria 07.1.04 del capítulo VII del Reglamento General de Normas Básicas de Seguridad Minera (Silice en minas a cielo abierto)?

- SÍLICE

Concentración promedio diaria

Concentración promedio diaria máxima

$25 / \mathrm{Q} \mathrm{mg} / \mathrm{m}^{3}$

En donde $\mathrm{Q}=$ Porcentaje $\mathrm{SO}_{2}$ de polvo

$5 \mathrm{mg} / \mathrm{m}^{3}$

5. BOE 24-4-1986

6. BOE 27-1-1990 
Orden de 31 de octubre de 1984, por la que se aprueba el Reglamento de trabajos con Amianto

Orden de 7 de enero de 1987, por la que se establecen normas complementarias al Reglamento sobre trabajos con riesgo de Amianto".

Orden de 26 de julio de 1993, por la que se modifican los artículos $2^{\circ}, 3^{\circ}$ y $13^{\circ}$ de la Orden de 31 de octubre de 1984, por la que se aprueba el Reglamento sobre trabajos con riesgo de Amianto, y el artículo $2^{\circ}$ de la Orden de 7 de enero de 1987, por la que se establecen normas complementarias al citado reglamento" ".

- AMIANTO

Crocidolita prohibida la utilización

Crisotilo '

Concentración promedio diaria

0.60 fibras $/ \mathrm{cm}^{4}$

Restantes variedades puras o en

mezclas, aún conteniendo Crisotilo

Concentración promedio diaria

0.30 fibras $/ \mathrm{cm}^{4}$

Por último, el Real Decreto 665/1997, de 12 de mayo, sobre la protección de los trabajadores contra los riesgos relacionados con la exposición a agentes cancerigenos durante el trabajo" ${ }^{\prime}$. regula el empleo de aquellas sustancias que están clasificadas como cancerígenos, mutagénicos o tóxicos para la reproducción, según el R.D. 363/199512, ordenándolas en doss categorías.

Las sustancias calalogadas como cancerigenos mulagénicos o tóxicos para la reproducción en la categoria 1 y 2 no tienen asignado ninglin limite va que no debe existir exposición a las mismas, y cuando sea necesario trabajar con ellas se establecerán los procedimientos de trabajo y las medidas de protección para evitar toda exposición.

7. BOE 30-10-1991

8. BOE 7-11-1984

9. BOE 15-1-1987

10. BOE 5-8-1993

11. BOE 24-5-1997

12. BOE 2-6-1995 y 5-6-1995 
Si valoramos la legislación con la que contamos nos encontramos con diversos problemas:

1. El análisis de los fundamentos y contenidos del Anexo II del RAMINP es bastante negativo:

a) Los valores límite se establecieron seguin el nivel de conocimiento que, sobre la toxicología de las sustancias, se tenía en fechas anteriores a 1962, con lo que en la mayoria de los casos han quedado obsoletos.

b) Fija unos valores limite como CMP (Concentración máxima permitida, actualmente los valores techol respondiendo a un criterio vigente en 1962. pero está demostrado que la valoración del riesgo está correlacionada con CPP rConcentración promedio permisible, es decir, la concentración a la que pueden estar expuestos la mayoria de los trabajadores durante un tiempo de 8 horas/dia o 40 horas/semana en el ambiente de trabajo), para la mayor parte de las sustancias.

c) Algunas unidades de medida están desfasadas. Ios límites para el "polvo industrial en suspensión" están definidos en millones de particulas por metro cúbicos. Según los conocimientos de 1962, el riesgo estaba en el número de pariculas que ingresaban en los alvéolos pulmonares. Desde hace mucho tiempo se sabe que el riesgo es función de la masa de polvo y no solo del número de partículas. y por tanto se evalúa en $\mathbf{m g} / \mathbf{m}^{3}$.

d) Por último, indicar que para muchas sustancias habitualmente empleadas en lugares de trabajo hoy en dia. no hay valores límite. ya que en 1962 no existían estudios toxicológicos o epidemiológicos sobre ellas, 0 incluso no se encontraban en el mercado.

2. En la actualidad contamos con la suficiente bibliografia científica como para que se admitiese la modificación del valor límite del Benceno, pero además al haberse demostrado que es un agente cancerígeno de categoria 1, el uso del mismo en los ambientes de trabajo debe restringirse al máximo. Por todo ello, el uso del Benceno en el trabajo debe regularse según el R.D. 665/1997 y no por la O. de 14 de septiembre de 1959.

3. Aunque el resto de los reglamentos son más modernos, y por lo tanto, los valores limite que aparecen en ellos no se encuentran tan desfasados, nos encontramos con la problemática de la velocidad a la que avanzan los conocimientos científicos, en temas de salud laboral, frente a la inercia que presenta la Administración a la modificación de la legislación vigente. 


\subsubsection{Directivas Europeas}

Una vez estudiado el panorama de la legislación interna española en cuanto a valores límite debemos señalar que la Comunidad Europea tiene publicadas diversas Directivas sobre estos valores.

En la DIRECTIVA 88/642/CEE ${ }^{13}$, de 16 de diciembre de 1988, por la que modifica la DIRECTIVA 80/1107/CEE sobre protección de los trabajadores contra los riesgos relacionados con la exposición a agentes químicos, físicos y biológicos. En el Anexo II bis, nos define valor límite como: la concentración media de la exposición, durante un período de 8 horas, de una sustancia en forma de gas, de vapor o de materia en suspensión en el aire en el lugar de trabajo.

También nos indica en que unidades debe expresarse los valores límite en la Comunidad Europea:

Para los gàses y vapores en $\mathrm{ml} / \mathrm{m}^{3}$ (ppm), valor independiente de las variables de temperatura y presión atmosférica, así como en $\mathrm{mg} / \mathrm{m}^{3}$ para una temperatura de $20^{\circ} \mathrm{C}$ y una presión de $101,3 \mathrm{kPa}$, valor que depende de las citadas variables.

El valor límite para las materias en suspensión se expresará en $\mathrm{mg} / \mathrm{m}^{3}$ para las condiciones de producción en el lugar de trabajo.

La DIRECTIVA 98/24/CE ${ }^{14}$, del 7 de abril de 1998, relativa a la protección de la salud y la seguridad de los trabajadores contra los riesgos relacionados con los agentes químicos durante el trabajo, nos define:

VALOR LÍMITE DE EXPOSICIÓN PROFESIONAL: El límite de la concentración media ponderada cronológicamente de un agente químico en el aire dentro de la zona de respiración de un trabajador con relación a un período de referencia específico.

Según esta Directiva, a trasponer antes del 5 de mayo del 2001, los Estados miembros establecerán un valor límite de exposición profesional nacional para todo agente químico que tenga fijado un valor límite indicativo de exposición profesional. Se podrán fijar valores límite de exposición profesional vinculantes a escala comunitaria que reflejarán todos los factores tenidos en cuenta con los valores límite indicativos y factores de viabilidad. Los estados miembros podrán establecer valores limite de exposición profesional nacionales basados en los vinculantes pero sin rebasarlos.

13. DOCE L356 24-12-1988

14. DOCE L131 5-5-1998 
Los términos "indicativo" y "vinculante", se emplean en esta Directiva para expresar los conceptos de "valor de referencia" y "valor límite".

Previamente la Comunidad habia publicado dos Directivas con valores límite indicativos:

- DIRECIIVA 91/322/CEE15, de 29 de mayo de 1991, relativa al establecimiento de valores limite de carácter indicativo mediante la aplicación de la DLRECTIVA 80/1107/CEE sobre protección de los trabajadores contra los riesgos relacionados con la exposición a agentes quí. micos.

- DIRECTIVA 96/94/CEE ${ }^{16}$, de 18 de diciembre de 1996, relativa al establecimiento de una segunda lista de valores limite de caracter indicativo mediante la aplicación de la DIRECTIVA 80/1107/CEE sobre protección de los trabajadores contra los riesgos relacionados con la exposición a agentes químicos, fisicos y biológicos.

- Actualmente existe un proyecto para elaborar la tercera Directiva.

Entre las clos listas contamos con un total de 50 valores limite "indicativos".

Los mayores inconvenientes que presenta son:

1. Que su transposición no es obligatoria por parte de los países miembros, tan solo indica unos valores máximos para que cada pais luego haga sus propias listas de valores límite, siempre que estos sean iguales o más restrictivos que los de las Directivas y presentando pruebas toxicológicas que lo avalen

2. Las sustancias que aparecen en dichas listas son poco frecuentes en los lugares de trabajo.

Por todo ello no son unas listas muy empleadas. 
2.1.3. Documento sobre límites de exposición profesional para Agentes Químicos en España

En el primer cuatrimestre de 1999, el I.N.S.H.T.(Instituto Nacional de Seguridad e Higiene en el Trabajo. Ministerio de Trabajo y Asuntos Sociales) ha publicado un documento en el que se establecen límites de exposición profesional indicativos para agentes químicos en España.

En dicho documento nos aparece la lista de valores límite más exhaustiva publicada hasta en momento por un organismo oficial español. Son valores de referencia, pero al derivar la misma del artículo 5 del Real Decreto 39/1997, de 17 de enero, por el que se aprueba el reglamento de los Servicios de Prevención 17: "cuando la evaluación exija la realización de mediciones, análisis o ensayos y la normativa no indique o concrete los métodos que deben emplearse, o cuando los criterios de evaluación contemplados en dicha normativa deban ser interpretados o precisados a la luz de otros criterios de carácter técnico, se podrán utilizar si existen, los métodos o criterios recogidos en:
a Normas UNE
b Guías del I.N.S.H.T. y otros organismos públicos
c Normas Internacionales
d Guías de entidades de reconocido prestigio"

son criterios a aplicar en el caso de no quedar cubiertos con la legislación vigente. Hemos de insistir en que son valores con carácter de recomendación y tan solo se deben emplear como una referencia técnica. No son valores legales nacionales.

Esta lista será ampliada y revisada anualmente por parte del comité técnico que la ha confeccionado. Basándose siempre en los criterios de la Unión Europea para la adopción de límites de exposición, los nuevos avances científicos y la evolución del marco legal.

Los límites de exposición profesional para agentes químicos, en el aire, se llamarán VALORES LIMITE AMBIENTALES (VLA). Se contempla también, en dicho documento la lista de indicadores biológicos de la exposición, que reciben el nombre de VALORES LIMITE BIOLÓGICOS (VLB).

Los VILA son valores de referencia para las concentraciones de los agentes químicos en el aire, y representan condiciones en las que, con los conocimientos actuales, se cree que la mayoría de los trabajadores pueden estar expuestos 8 horas diarias y 40 semanales, durante toda su vida laboral, sin sufrir efectos adversos para su salud. 
Según el tipo de exposición, siempre haciendo referencia a la vía respiratoria, tendremos dos límites diferentes:

\section{- VLA-ED. Valor Limite Ambiental - Exposición Diaria.}

Es la concentración media del agente químico en la zona de respiración del trabajador medida para una jornada laboral real y referida a una jornada estándar de 8 horas diarias.

\section{- VLA-EC. Valor Límite Ambiental - Exposición de Corta Duración.}

Es la concentración media del agente químico en la zona de respiración del trabajador medida o calculada para cualquier período de 15 minutos a lo largo de la jornada laboral, excepto para aquellos agentes químicos para los que se especifique un período de referencia inferior, en la lista de valores límite.

\subsubsection{Criterios de referencia internacionales}

Existen diferentes organizaciones científicas de referencia pero la de mayor prestigio en el mundo de la Higiene Industrial es la ACGIH (American Conference of Governmental Industrial Hygienist). Es una asociación de higienistas industriales que trabajan en instituciones del gobierno norteamericano.

Los valores para agentes químicos en el ambiente de trabajo que establece son los TLV "Threshold limit value" y se basan exclusivamente en criterios técnicos. Tan solo son valores recomendados pero son los que gozan de un mayor prestigio internacional. Es la lista con mayor número de sustancias y es revisada anualmente.

El TLV (en español valor límite umbral), es una marca registrada por la ACGHH, se refiere a la concentración del agente químico en el aire del ambiente de trabajo a la que se supone que puede estar expuestos casi todos los trabajadores, de forma repetitiva, día tras día, sin efectos nocivos. Los TLV pueden ser de tres tipos:

- TLV-TWA. Valor limite umbral - Media ponderada en el tiempo.

Concentración media ponderada en el tiempo, para una jornada laboral de 8 horas y $\mathbf{4 0}$ horas semanales, a la cual la mayoría de los trabajadores pueden estar expuestos repetidamente día tras día sin sufrir efectos adversos.

- TLV-C. Valor limite umbral - Valor techo.

Concentración que no debería ser pasada en ningún instante. Para algunas sustancias como los gases irritantes quizás solo sea adecuada la categoría de TiV-C. 


\section{- TLV-STEL. Valor límite umbral - Límite de exposición para cortos perí- odos de tiempo.}

Concentración a la que pueden estar expuestos los trabajadores durante un corto espacio de tiempo sin sufrir irritación, daño crónico o irreversible en los tejidos o narcosis en grado suficiente para aumentar la probabilidad de lesiones accidentales, dificultar salir por si mismo de una situación de peligro o reducir sustancialmente la eficacia en el trabajo, y siempre que no se sobrepase el TLV-TWA. No es un límite de exposición separado e independiente sino un complemento de la media ponderada en el tiempo (TWA) cuando se admite la existencia de efectos agudos de una sustancia cuyos efectos tóxicos son, primordialmente de carácter crónico.

Se define como la exposición media ponderada en el tiempo durante 15 minutos que no debe sobrepasarse en ningún momento de la jornada, aunque la media ponderada en el tiempo durante las 8 horas sea inferior al TLV-TWA.

\section{2. ÍNDICES DE EXPOSICIÓN BIOLÓGICA}

La exposición profesional a sustancias tóxicas que se encuentren en el ambiente, se ha venido determinando mediante análisis químicos del aire del puesto de trabajo. Siendo el método que presenta mayores ventajas, no es el ideal, ya que no puede brindarnos una información completa clel nivel real de exposición del trabajador. Esto puede deberse a que:

- Hay agentes que se absorben por diferentes vías.

- El trabajador puede estar expuesto a esa sustancia fuera del ambiente de trabajo.

- Se producen efectos combinados por exposiciones múltiples, etc.

Por todo ello se realiza el control biológico de los agentes químicos, con el que también se vigila el estado de salud de los trabajadores.

Estos índices representan los niveles de alerta de la respuesta biológica a una compuesto químico o alguno de sus metabolitos en tejidos, fluidos biológicos o aire expirado de los trabajadores expuestos, independientemente de la vía de entrada de dicho compuesto al organismo.

En la legislación española encontramos un solo ejemplo de aplicación conjunta de la evaluación biológica y ambiental en el Reglamento del Plomo (Orden de 9 de abril de 1986), donde se establece un valor ambiental y otro biológico para definir al "trabajador expuesto", un valor ambiental como nivel de acción, y un valor límite ambiental y biológico. 
En la Directiva 98/24/CE nos define como Valor Límite Biológico: el límite de la concentración en el medio biológico adecuado del agente químico de que se trate, su metabolito u otro indicador de efecto.

Como comentamos en el apartado 2.1.3, el I.N.S.H.T. ha publicado un documento en el que nos define los futuros Valores Límites Biológicos (VLB), como valores de referencia para los Indicadores Biológicos asociados a la exposición global a los agentes químicos. Estos valores no deben emplearse como medida de efectos adversos ni en el diagnóstico de enfermedades profesionales, son un complemento del control ambiental.

A medida que vayan estableciéndose los distintos VLB, se irán publicando en el citado documento.

Como criterios de referencia, en el caso de no contar con índices de exposición biológica legales o de referencia españoles, pueden emplearse los de la A.C.G.I.H., que desde 1985 publica conjuntamente con la lista de TLVs, una lista de límites biológicos, denominándolos Biological Exposure Indices (BEIs).

- Ventajas de la eValuación BIOLÓGICA.

1. Informa de la exposición global. Laboral y extralaboral.

2. Evaluación del tóxico por cualquier vía de entrada.

3. Detecta hipersusceptibilidades individuales.

4. Pone de manifiesto hábitos de trabajo.

5. Integra factores como la fatiga, estrés, etc.

- INCONVENIENTES DE LA EVALUACIÓN BIOLÓGICA.

1. Utiliza como elemento de muestreo al ser humano.

2. Aplicable tan solo a tóxicos cuya vida media $>5 \mathrm{~h}$.

3. Sólo puede emplearse en colectivos homogéneos.

4. Desconocimiento de valores de referencia de un gran número de sustancias.

5. Exclusión de sensibilizantes, irritantes y cancerígenos.

6. Falta de normalización en las técnicas analíticas.

Nos encontramos como principal inconveniente con la falta de criterios legales para los diversos agentes químicos, por lo que la mayoría de las veces los empleamos como índices orientativos de la posible intoxicación del trabajador por contaminación en el lugar de trabajo. 


\section{CRITERIOS de valoración para agentes físicos}

Los agentes físicos que pueden producir enfermedades laborales en el hombre se presentan como diferentes formas de energía que actúan sobre el organismo.

Tenemos diversas clases de energía: ondas que transmiten el sonido (ruido y vibraciones), las radiaciones ionizantes y no ionizantes, entre ellas la energía luminosa y el calor. Para todas ellas contamos con criterios de valoración con los que comparar los valores obtenidos en las mediciones en el lugar de trabajo, muchos de ellos vienen reflejados en la legislación vigente y para otros hemos de aplicar los criterios indicados por la bibliografía científica.

Estos criterios, al igual que en el caso de los agentes químicos, están basados en el daño que producen en el organismo humano y en el porcentaje de población expuesta que queremos proteger.

\subsection{RUIDO}

La problemática que puede generar los diferentes niveles de ruido a los que se encuentra sometido el trabajador está tipificada en el Real Decreto 1316/1989, de 27 de octubre, sobre protección de los trabajadores frente a los riesgos derivados de la exposición al ruido durante el trabajo ${ }^{18}$.

En este reglamento se acotan cuatro niveles de actuación, que actúan como criterios de valoración a la hora de contrastar el ruido al que se encuentra sometido el trabajador. En cada uno de ellos a medida que aumenta el valor del nivel aumentan las exigencias de protección de los trabajadores sometidos a ellos.

- Como parámetro indicador del nivel de ruido emplea el concepto de Nivel Diario Equivalente (LA eq, d), es decir, el nivel de presión acústica continuo equivalente, ponderado $A$, al que está sometido el trabajador durante 8 horas al día.

1) Trabajadores sometidos a niveles de ruido diario equivalente (LA eq, $d$ ) inferior a $80 \mathrm{~dB}(\mathrm{~A})$. En este caso no han de aplicarse ningún tipo de medidas correctoras o de protección.

2) Trabajadores sometidos a niveles de ruido diario equivalente (LA eq, d) entre $80 \mathrm{~dB}$ (A) y $85 \mathrm{~dB}$ (A).

Se llevará a cabo una nueva evaluación de los niveles de ruido cada tres años. Deberán realizarse controles médicos auditivos cada cinco años.

18. BOE 2-11-1989, corrección de errores 9-12-1989, 26-5-1990 
El empresario pondrá a disposición de los trabajadores los equipos de protece ción personal.

El empresario deberá formar e informar a los trabajadores sobre los niveles de ruido a los que está sometido.

3) Trabajadores sometidos a niveles de ruido diario equivalente (LA eq, d): entre $85 \mathrm{~dB}$ (A) y $90 \mathrm{~dB}$ (A).

Se llevará a cabo una nueva evaluación de los niveles de ruido cada año.

Deberán realizarse controles médicos auditivos cada tres años.

El empresario proporcionará a los trabajadores los equipos de protección personal.

El empresario deberá formar e informar a los trabajadores sobre los niveles de ruido a los que está sometido.

4) Trabajadores sometidos a niveles de ruido diario equivalente (LA eq, d) superior a $90 \mathrm{~dB}$ (A) y niveles de pico superiores a $140 \mathrm{~dB}$ (A).

Se llevará a cabo una nueva evaluación de los niveles de ruido cada año.

Deberán realizarse controles médicos auditivos cada año.

El empresario aplicará medidas de protección colectiva siempre que sea técnicamente posible u obligará a los trabajadores a utilizar los equipos de protección personal.

El empresario deberá formar e informar a los trabajadores sobre los niveles de ruido a los que está sometido.

Se señalizarán los puestos o zonas de trabajo según el R.D. 485/1997.

\subsection{VIBRACIONES}

Los efectos producidos por las vibraciones en el organismo son conocidos desde antiguo, y aunque no contamos con legislación sobre el tema, si están recogidos como enfermedad profesional diversos síndromes y enfermedades producidas en el hombre por el uso de herramientas que transmiten vibraciones.

Debido a todo ello, nos encontramos que tan solo contamos con Normas Internacionales ISO y ANSI, para su evaluación y con los criterios de valoración de organismos internacionales de reconocido prestigio, en este caso de la A.C.G.I.H., como valores de referencia.

Los valores límites, propuestos por la A.C.G.I.H., son para vibraciones Mano Brazo, en el caso de emplear herramientas manuales, y para vibraciones mecánicas inducidas de cuerpo entero, en el caso de conductores de vehículos (camiones, tractores, etc.). También en este caso se llaman TLV y son niveles de aceleración medidos en $\mathrm{m} / \mathrm{s} 2$. 


\subsection{RADIACIONES IONIZANTES}

El trabajo con equipos que emitan radiaciones ionizantes, debido a su potencial peligrosidad como inductores de cáncer en humanos, ha sido regulado mediante diversos reglamentos, siendo el Real Decreto 53/1992, de 24 de enero, por el que se aprueba el Reglamento sobre Protección Sanitaria contra radiaciones ionizantes ${ }^{19}$, en el que aparecen los criterios de valoración para controlar los niveles de exposición.

En él se señalan los límites de dosis efectiva para trabajadores expuestos y para el público, no teniendo en cuenta las dosis recibidas del fondo radioactivo natural y de la exposición sufrida como consecuencia de exámenes y tratamientos médicos.

\begin{tabular}{|l|c|c|}
\hline \multicolumn{3}{|c|}{ LIMITES ANUALES DE DOSIS } \\
\hline EXPOSICION TOTAL & $\begin{array}{c}\text { TRABAJADORES } \\
\text { EXPUESTOS } \\
\text { mSv }\end{array}$ & $\begin{array}{c}\text { MIEMBROS } \\
\text { DEL PÚBLICO } \\
\text { mSv }\end{array}$ \\
\hline DOSIS EFECTIVA & 50 & 5 \\
\hline CRISTALINO & 50 & 5 \\
\hline PIEL & 150 & 15 \\
\hline MANOS, BRAZOS, PIES O TOBILLOS & 500 & 50 \\
\hline ORGANOS O TEJDOS & 500 & 50 \\
\hline MUJERES EN PERIODO FÉRTIL & 500 & 50 \\
\hline ABDOMEN & 13 & - \\
\hline MUJERES GESTANTES & 10 & - \\
\hline OPERACIONES ESPECIALES & Máximo doble del límite & \\
\hline
\end{tabular}

19. BOE 12-2-1992 
Los límites de dosis para estudiantes que manejen fuentes radioactivas en fun:ción de sus estudios son iguales que para los trabajadores profesionalmente expuestos en el caso de que tengan 18 años o más. Si son mayores de 16 y menores de 18 años el límite de exposición será tres décimos de los límites establecidos para los trabajadores profesionalmente expuestos.

\subsection{RADIACIONES NO IONIZANTES}

Dentro de las radiaciones no ionizantes merece un tratamiento a parte la radiación en el espectro visible: la iluminación.

Este tipo de radiaciones se diferencian de la ionizantes en que su poder energético no es suficiente para ionizar las estructuras atómicas o moleculares, por lo. que se convierten al incidir sobre el organismo en energía rotacional, vibracional o capaz de modificar configuraciones electrónicas, es decir, un aumento de energía cinética y producción de calor.

Salvo para la radiación en el espectro de luz visible no hay ninguna legislación española que nos indique valores límite para el resto de las radiaciones del espectro: Ultravioleta, Infrarrojo, Microondas y Radiofrecuencias, Láser y Ultrasonidos.

Debido a esta ausencia de legislación se siguen criterios de valoración de diferentes organismos internacionales así como de organismos de reconocido prestigio: científico (A.C.G.I.H.).

La existencia de una propuesta de Directiva relativa a agentes físicos, nos induce a pensar en que contaremos en un futuro con normativa legal en la que aparezcan criterios de valoración de la exposición a radiaciones no ionizantes.

En el caso de las radiaciones ultravioleta tenemos los criterios emitidos por la Asociación Internacional de Protección contra Radiaciones (I.R.P.A./I.N.I.R.C.) y la Organización Mundial de la Salud (O.M.S.). Son límites de protección bastante complejos de aplicar ya que el umbral de daño varía con la longitud de onda, el tiempo de exposición y otros factores.

Estos criterios coinciden con los adoptados por la A.C.G.I.H. para radiaciones: ultravioleta. También cuenta con valores límite para radiaciones en la zona del vist: ble e infrarrojo.

Para las radiaciones de microondas el I.R.P.A./I.N.I.R.C. y el American National Standards Institute (A.N.S.I.) proponen un sistema de límites de exposición a radiaciones electromagnéticas de frecuencias comprendidas eñtre 100 y 300 GHz. Para ello tienen en cuenta factores físicos ligados a la radiación incidente, factores ligados a la exposición y otros ligados a las características biológicas.

La magnitud que empleamos para medirlas es la energía absorbida por unidad de tiempo y masa (DAS - Debit d'Absorption Spécifique) y se expresa en $\mathrm{W} / \mathrm{kg}$. 
Para el público en general se ha incorporado un factor adicional de seguridad igual a 5 sobre los límites fijados para los trabajadores y al fin de que pueda estar expuesto $24 \mathrm{~h}$. al día durante períodos prolongados.

La A.C.G.I.H., fija los valores máximos de exposición bajo condiciones a las cuales casi todos los trabajadores pueden estar expuestos sin efectos adversos durante la jornada laboral.

Para las radiaciones emitidas por láseres pulsados tenemos dos criterios de valoración diferentes. La A.N.S.I. basa los criterios en condiciones de exposición agudas, mientras que la A.C.G.I.H., lo hace fijando la exposición bajo condiciones a las cuales casi todos los trabajadores pueden estar expuestos sin efectos adversos durante la jornada laboral.

\subsubsection{Iluminación}

Los valores límite que se manejan en Higiene Industrial para la energía luminosa vienen reflejados en el Real Decreto 486/1997, de 14 de abril, por el que se establecen las disposiciones mínimas en seguridad y salud en el lugar de trabajo $^{20}$, en su ANEXO IV.

Los criterios que han servido para establecer estos valores límite de iluminación mínimos tienen en cuenta la actividad llevada a cabo en el centro de trabajo y las exigencias visuales para el desarrollo de las diferentes tareas.

\begin{tabular}{|l|c|}
\hline $\begin{array}{l}\text { ZONA O PARTE DEL } \\
\text { LUGAR DE TRABAJO }\end{array}$ & $\begin{array}{c}\text { NIVEL MINIMO DE ILUMINACION } \\
\text { (Lux) }\end{array}$ \\
\hline Zonas donde se ejecutan tareas: & 100 \\
- Bajas exigencias visuales & 200 \\
- Exigencias visuales moderadas $\quad$ Exigencias visuales altas & 500 \\
- Exigencias visuales muy altas & 1000 \\
Áreas o locales de uso ocasional & 50 \\
Areas o locales de uso habitual & 100 \\
Vías de circulación de uso ocasional & 25 \\
Vias de circulación de uso habitual & 50 \\
\hline
\end{tabular}

20. BOE 23-4-1997 
Estos niveles deberán duplicarse cuando concurran una serie de circunstancias:

I. Cuando por las características de la zona o lugar de trabajo existan riesgos: apreciables de caídas, choques $u$ otros accidentes.

II. En las zonas de trabajo, cuando un error de apreciación visual pueda suponer: - un peligro para el trabajador en la tarea que ejecuta,

- o un peligro para terceros,

- o cuando el contraste de luminancias o color entre el objeto a visualizar y el fondo sobre el que se encuentra sea muy débil.

\subsection{TEMPERATURA}

Al igual que los niveles de iluminación, los valores de confort o condiciones ambientales en los lugares de trabajo, nos vienen legislados en el Real Decreto 486/1997, de 14 de abril, por el que se establecen las disposiciones minimas en seguridad y salud en el lugar de trabajo, en su ANEXO III.

\begin{tabular}{|l|c|c|c|}
\hline & \multicolumn{2}{|c|}{ TRABAJOS SEDENTARIOS } & TRABAJOS LIGEROS \\
\hline & $\begin{array}{c}\text { AMB. NO } \\
\text { CALUROSO }\end{array}$ & $\begin{array}{c}\text { AMB. } \\
\text { CALUROSO }\end{array}$ & $\begin{array}{c}\text { AMB. } \\
\text { CALUROSO }\end{array}$ \\
\hline TEMPERATURA & $17-27^{\circ} \mathrm{C}$ & $17-27^{\circ} \mathrm{C}$ & $14-25{ }^{\circ} \mathrm{C}$ \\
\hline HUMEDAD RELATIVA & $30-70 \%$ & $30-70 \%$ & $30-70 \%$ \\
\hline $\begin{array}{l}\text { CORRIENTES CON } \\
\text { AIRE ACONDICIONADO }\end{array}$ & $0.25 \mathrm{~m} / \mathrm{s}$ & $0.35 \mathrm{~m} / \mathrm{s}$ & $0.35 \mathrm{~m} / \mathrm{s}$ \\
\hline RENOVACIONES DE AIRE & & & \\
\hline POR TRABAJADOR & $30 \mathrm{~m} 3 / \mathrm{h}$ & $50 \mathrm{~m} 3 / \mathrm{h}$ & $50 \mathrm{~m} 3 / \mathrm{h}$ \\
\hline
\end{tabular}

Estos valores establecidos por la legislación vigente no son aptós para trabajos con un gran esfuerzo físico o trabajadores sometidos a temperaturas extremas. Paŕa estas circunstancias hemos de emplear valores limites de referencia existentes en la bibliografía científica. 
La A.C.G.I.H. propone dos criterios de valoración para condiciones de temperatura extrema:

- Para condiciones de estrés por calor emplea el índice W.B.G.T.(Wet Bulb Globe Temperature). Los valores límite obtenidos mediante este índice hacen referencia a las condiciones de estrés por calor a las que se cree que casi todos los trabajadores pueden estar expuestos repetidamente sin efectos adversos para la salud, siempre que se les someta a una acondicionamiento previo.

- Para temperaturas extremas por frío establece una serie de TLV en función de la temperatura externa y la velocidad del aire (viento), para proteger a los trabajadores de los efectos más graves tanto del estrés por frío (hipotermia), como de las lesiones causadas por el frío.

\section{Criterios de valoración para agentes biológicos}

El Real Decreto 664/1997, de 12 de mayo, sobre protección a los trabajadores contra los riesgos relacionados con la exposición a agentes biológicos durante el trabajo ${ }^{21}$, no nos señala ningún valor límite para la exposición a agentes biológicos, por lo que no contamos con ninguna referencia legal, tan solo nos indica en su artículo 6 siempre que sea posible deberá evitarse la exposición a los mismos y cuando no sea técnicamente posible, se reducirá el nivel de exposición al nivel más bajo posible para garantizar adecuadamente la salud y seguridad de los trabajadores afectados.

\section{CONCLUSIONES}

Una vez hemos expuesto los distintos criterios de valoración existentes para los agentes químicos, físicos ó biológicos, nos encontramos ante una disyuntiva a la hora de aplicarlos en las empresas en las que ha de corregirse una problemática que puede inducir una enfermedad profesional en el personal que trabaja en ella.

I. Como dijimos anteriormente, para los agentes químicos aplicamos la legislación vigente en todos los casos posibles, pero la lista legal con mayor número de valores se encuentra totalmente desfasada con respecto a los criterios científicos que se barajan hoy en día. Por ello, para proteger la salud

21. BOE 24-5-199 
de estos trabajadores, es bastante frecuente, hasta hoy, el empleo de los valores límite propuestos por instituciones científicas internacionales de: reconocida solvencia por parte de los técnicos de prevención, la Administración y las organizaciones sindicales. Pero a raíz, de la publicación. de la lista de valores límite de referencia, editada por el I.N.S.H.T., y siguiendo los planteamientos del R.D. 39/1997, art. 5, ésta pasa a ser el segundo criterio de valoración para agentes químicos en el caso de no contar con legislación vigente. Planteamiento muy adecuado desde el punto de vista preventivo, pero que puede presentar un inconveniente a la hora de su aplicación:

El administrado y responsable de cumplir con dichos valores, tiene derecho a exigir que se le aplique la legislación vigente, aunque esta sea menos restrictiva que los valores límite publicados, anualmente, por el I.N.S.H.T.

Esta situación puede provocar dificultades a los profesionales en el establecimiento de objetivos aceptables por la empresa y trabajadores. Desde el punto de vista operativo, la solución se debe estudiar individualmente y en función de la situación de partida de cada empresa.

II. En el caso de los agentes físicos, contamos con una legislación más reciente y por tanto adaptada a criterios científicos más avanzados, aunque en los casos en los que carecemos de normativa legal han de aplicarse los valores límite de referencia.

III. Por último, con los agentes biológicos la legislación es relativamente reciente, fruto de una directiva de 1990 , y por tanto científicamente presenta un menor desfase. 
6. Bibliografía

(1) DOCUMENTO SOBRE LÍMITES DE EXPOSICIÓN PROFESIONAL PARA AGENTES QUÍMICOS EN ESPAÑA, I.N.S.H.T., (1999).

(2) TLVs AND BEIs. THRESHOLD LIMIT VALUES FOR CHEMICAL SUBSTANCES AND PHISICAL AGENTS, American Conference of Governmental Industrial Hygienist, (1998).

(3) GONZÁLEZ FERNÁNDEZ, E., Los límites personales de exposición laboral, Mapfre Seguridad, (24), 47-55, (1986).

(4) BARTUAL SÁNCHEZ, J. y col., Criterios de valoración en higiene industrial, N.T.P. $\mathrm{n}^{\circ}$ 244, I.N.S.H.T., (1989).

(5) MORTON LIPPMANN, Ph. D., Environmental toxicology and exposure limits for ambient air, Appl. Occup. Environ. Hyg., 8 (10), 847-858, (1993).

(6) HUNTER, W.J. et al., Occupational exposure limits for chemicals in the european union, Occup. Environ. Medicine, (54), 217-222, (1997).

(7) INICIATIVAS EUROPEAS ORIENTADAS A ESTABLECER VALORES LÍMITE DE EXPOSICIÓN, Prevención Express, 279, 8-9, (1998).

(8) INDICADỚRES BIOLÓGICOS DE EXPOSICIÓN, Grupo Científico para la Vigilancia de Atmósferas de Trabajo, Cahiers de Notes Documentaires. $n^{\circ} 150$, (1993).

(9) НUICI MONTAGUD, A., Control biológico de contaminantes químicos, N.T.P. $\mathrm{n}^{\circ} 146$, I.N.S.H.T., (1985).

(10) LANAS UGARTEBURU, P.M., Conocimiento, evaluación y control del ruido, A.P.A., (1996).

(11) HERAS COBO, C., Factores ergonómico-ambientales del trabajo en interiores, Diputación General de Aragón, (1996). 